\title{
Entanglement and the Quantum Brachistochrone Problem
}

\author{
A. Borras ${ }^{1}$, C. Zander ${ }^{2}$, A.R. Plastino ${ }^{1,2,3 *}$, M. Casas $^{1}$, and A. Plastino ${ }^{3}$ \\ ${ }^{1}$ Departament de Física and IFISC-CSIC, Universitat de les Illes Balears, 07122 Palma de Mallorca, Spain \\ ${ }^{2}$ Physics Department, University of Pretoria, Pretoria 0002, South Africa \\ ${ }^{3}$ National University La Plata-CONICET, C.C. 727, 1900 La Plata, Argentina
}

(Dated: February 2, 2008)

\begin{abstract}
Entanglement is closely related to some fundamental features of the dynamics of composite quantum systems: quantum entanglement enhances the "speed" of evolution of certain quantum states, as measured by the time required to reach an orthogonal state. The concept of "speed" of quantum evolution constitutes an important ingredient in any attempt to determine the fundamental limits that basic physical laws impose on how fast a physical system can process or transmit information. Here we explore the relationship between entanglement and the speed of quantum evolution in the context of the quantum brachistochrone problem. Given an initial and a final state of a composite system we consider the amount of entanglement associated with the brachistochrone evolution between those states, showing that entanglement is an essential resource to achieve the alluded time-optimal quantum evolution.
\end{abstract}

PACS numbers: 03.65.Xp, 03.65.Ca, 03.67.Lx, 03.67.Hk

Keywords: Time-Optimal Quantum Evolution, Quantum Brachistochrone Problem, Quantum Entanglement.

The celebrated brachistochrone problem has played a distinguished role in the history of mechanics [1]. A quantum dynamical optimization problem akin to the classical brachistochrone one has attracted the attention of several researchers in recent years [2, 3, 4, 5]. This problem deals with the Hamiltonian generating the optimal quantum evolution $|\psi(t)\rangle$ (in the sense of the one requiring the shortest time $\tau$ ) between two prescribed states $\left|\psi_{I}\right\rangle$ and $\left|\psi_{F}\right\rangle$. It has been recently established that the entanglement features of quantum states are important in connection with dynamical optimization problems [6, 7, 8, ]. Previous research done on this subject has been focused upon the study of the time needed to reach an orthogonal state by systems evolving under a given Hamiltonian [10]. The details of these kind of studies depend strongly on the particular Hamiltonian considered, and each case requires a separate treatment (see, for instance, $[8,9]$ ). The aim of the present contribution is to revisit the connection between entanglement and optimal evolution from the different (but related) point of view provided by the quantum brachistochrone problem. An important advantage of the brachistochrone approach is that it allows for a more general and unified investigation of the alluded connection, going beyond the separate analysis of individual cases.

\section{ROLE OF ENTANGLEMENT IN TIME-OPTIMAL QUANTUM EVOLUTION}

As already mentioned, our aim is to explore the connection between entanglement and the quantum brachistochrone evolution of a bipartite system constituted by two subsystems $A$ and $B$ (with associated Hilbert spaces

\footnotetext{
*Corresponding Author: arplastino@maple.up.ac.za
}

of dimensions $N_{A}$ and $N_{B}$, respectively). We want to quantify the amount of entanglement involved when implementing the evolution of an initial state $\left|\psi_{I}\right\rangle$ to a final state $\left|\psi_{F}\right\rangle$ in the shortest possible time. Following [3], we are going to consider the optimal evolution under the constraint that the difference between the maximum and minimum eigenenergies of the Hamiltonian generating the unitary transformation $\left|\psi_{I}\right\rangle \rightarrow\left|\psi_{F}\right\rangle=e^{\frac{i H \tau}{\hbar}}\left|\psi_{I}\right\rangle$ be less or equal to a given constant energy $2 \omega$. This constraint is imposed due to the following reason: if the differences between the eigenenergies of the Hamiltonian are arbitrarily large, then it is easy to implement a quantum evolution connecting the alluded states and taking a time $\tau$ that can be made arbitrarily small. The optimal time evolution is given by [3]

$$
\begin{aligned}
|\psi(t)\rangle= & {\left[\cos \left(\frac{\omega t}{\hbar}\right)-\frac{\cos \frac{1}{2} \theta}{\sin \frac{1}{2} \theta} \sin \left(\frac{\omega t}{\hbar}\right)\right]\left|\psi_{I}\right\rangle } \\
& +\frac{1}{\sin \frac{1}{2} \theta} \sin \left(\frac{\omega t}{\hbar}\right)\left|\psi_{F}\right\rangle .
\end{aligned}
$$

This expression satisfies $|\psi(0)\rangle=\left|\psi_{I}\right\rangle$ and $|\psi(\tau)\rangle=\left|\psi_{F}\right\rangle$, where

$$
\tau=\frac{\hbar \theta}{2 \omega} .
$$

To determine the parameter $\theta$, the final state $\left|\psi_{F}\right\rangle$ has to be written in the form

$$
\left|\psi_{F}\right\rangle=\cos \frac{1}{2} \theta\left|\psi_{I}\right\rangle+e^{i(\phi+\pi / 2)} \sin \frac{1}{2} \theta\left|\bar{\psi}_{I}\right\rangle,
$$

where $\left|\bar{\psi}_{I}\right\rangle$ is a state orthogonal to the initial state $\left|\psi_{I}\right\rangle$ contained in the two-dimensional subspace (of the full Hilbert space) spanned by the initial and final states. 
Since both $\left|\psi_{I}\right\rangle$ and $\left|\psi_{F}\right\rangle$ are specified, the values of $\phi$ and $\theta$ can be regarded as known, the latter being the angle of separation of these two states. We are going to focus upon the case where the initial and final states are orthogonal, thus having $\theta=\pi$ and,

$$
\begin{aligned}
\tau & =\frac{\pi \hbar}{2 \omega} \\
|\psi(t)\rangle & =\cos \left(\frac{\omega t}{\hbar}\right)\left|\psi_{I}\right\rangle+\sin \left(\frac{\omega t}{\hbar}\right)\left|\psi_{F}\right\rangle .
\end{aligned}
$$

From the point of view of the physics of information and computation this case is the most interesting because the evolution of a computer devise into a state orthogonal to the initial one can be identified with an elementary information processing step [11].

In order to assess how much entanglement is involved in the brachistochrone evolution we are going to compute the time-average of the entanglement $\mathcal{E}[\psi(t)]$ during the optimal time evolution from $\left|\psi_{I}\right\rangle \rightarrow\left|\psi_{F}\right\rangle$,

$$
\langle\mathcal{E}\rangle=\frac{1}{\tau} \int_{0}^{\tau} \mathcal{E}(t) \mathrm{d} t
$$

The idea of time averaged entanglement has been recently discussed by several researchers and was found to constitute a useful concept to study a variety of problems (see, for instance, [12] and references therein). To facilitate the computations we use the linear entropy as entanglement measure,

$$
\mathcal{E}[\psi(t)]=\frac{N_{A}}{N_{A}-1}\left[1-\operatorname{Tr}\left(\rho_{A}^{2}\right)\right],
$$

where $\rho_{A}$ is the marginal density matrix associated with one of the two subsystems (the one with the Hilbert space of lower dimension), $\rho_{A}=\operatorname{Tr}_{B}[\rho(t)]$, and the global density matrix describing the bi-partite system is $\rho(t)=|\psi(t)\rangle\langle\psi(t)|$. Since the entanglement $\mathcal{E}(t)$ depends upon time only through the quantity $\xi=\frac{\omega t}{\hbar}$, the integral giving the time-averaged entanglement can be put under the guise

$$
\langle\mathcal{E}\rangle=\frac{2}{\pi} \int_{0}^{\pi / 2} \mathcal{E}(\xi) d \xi,
$$

implying that $\langle\mathcal{E}\rangle$ is independent of $\omega$ and since $\tau=\frac{\pi \hbar}{2 \omega}$, this means that $\langle\mathcal{E}\rangle$ is also independent of the absolute time taken.

Highly asymmetric states can evolve optimally without entanglement. This is clearly illustrated by the extreme case corresponding to factorizable initial and final states of the form

$$
\left|\tilde{\psi}_{F}\right\rangle=\left|\phi_{2}\right\rangle \otimes\left|\phi_{0}\right\rangle,
$$

where one of the subsystems (in this case, the second subsystem) is in the same state $\left|\phi_{0}\right\rangle$ at the beginning and at the end of the process. It is plain that in such circumstances the brachistochrone evolution can be implemented without entanglement. Indeed, the optimal evolution is given by the time dependent, separable state

$$
|\tilde{\psi}(t)\rangle=|\phi(t)\rangle \otimes\left|\phi_{0}\right\rangle,
$$

$|\phi(t)\rangle$ being the optimal evolution of the first subsystem connecting the states $\left|\phi_{1}\right\rangle$ and $\left|\phi_{2}\right\rangle$. It is also clear that in this highly asymmetric setting only one of the subsystems (the first one) is evolving and, consequently, we are essentially dealing with the evolution of a single system. That is, the composite nature of the total system plays no role in an evolution like (9). This is fully consistent with similar results reported by Giovannetti, Lloyd and Maccone in [6]. There it was pointed out that, for composite a system with non-interacting subsystems, assymetric non-entangled states in which all the energetic resources are concentrated in one subsystem can saturate the fundamental lower bound for the time required to reach a state orthogonal to the original one. On the contrary, symmetric non-entangled states with evenly shared energetic resources do not saturate the alluded bound.

States of the form (8) are the only pairs of orthogonal initial and final states such that the time averaged entanglement of the concomitant optimal evolution vanishes. This can be seen as follows. Suppose that the time averaged entanglement is zero. That implies that the entanglement is zero at all times $t \in[0, \tau]$. In particular, the initial and the final states must be separable. Since they are also orthogonal we can assume, without loss of generality, that the initial and final states are, respectively, of the form $\left|\psi_{I}\right\rangle=|0\rangle \otimes\left|\phi_{r}\right\rangle$ and $\left|\psi_{F}\right\rangle=|1\rangle \otimes\left|\phi_{s}\right\rangle$. Now, the time dependent state must be separable also at all intermediate times $t \in[0, \tau]$. In particular, it must be separable at the time corresponding to $\frac{\omega t}{\hbar}=\frac{\pi}{4}$. That is, the state $(1 / \sqrt{2})\left(|0\rangle \otimes\left|\phi_{r}\right\rangle+|1\rangle \otimes\left|\phi_{s}\right\rangle\right)$ has to be separable. But for this state we have $\mathcal{E}=1-\left|\left\langle\phi_{r} \mid \phi_{s}\right\rangle\right|^{2}$. Consequently, the entanglement of this state will be zero only if $\left|\left\langle\phi_{r} \mid \phi_{s}\right\rangle\right|=1$, and so the pair of initial and final states is of the form (8).

\section{TWO-QUBIT SYSTEMS}

The features of the optimal evolution which are associated with the composite nature of the system (such as the role of entanglement) are more clearly seen when we study the evolution of symmetric states. For this reason we are now going to pay special attention on the optimal evolution of two-qubit systems associated with symmetric initial and final states.

First we are are going to consider three specific cases of optimal evolution of symmetric sates of two qubit sys-

$$
\left|\tilde{\psi}_{I}\right\rangle=\left|\phi_{1}\right\rangle \otimes\left|\phi_{0}\right\rangle
$$


tems associated, respectively, with the following three pairs of initial and final states,

- (i) $\quad|00\rangle \rightarrow \frac{\cos \alpha}{\sqrt{2}}\{|01\rangle+|10\rangle\}+\sin \alpha|11\rangle$

- (ii) $\frac{1}{\sqrt{2}}\{|01\rangle+|10\rangle\} \rightarrow \frac{1}{\sqrt{2}}\{|00\rangle+|11\rangle\}$

- (iii) $\frac{1}{\sqrt{2}}\{|00\rangle-i|11\rangle\} \rightarrow \frac{1}{\sqrt{2}}\{i|00\rangle-|11\rangle\}$.

Let us discuss each example separately:

(i). In this case we have the evolution of a separable state into one of a family of possible final states parameterized by the real parameter $\alpha$. According to the value of this parameter we may have for the final state either another separable state $\left(\alpha=\frac{\pi}{2}\right)$, an intermediately entangled state $\left(\alpha \in\left(0, \frac{\pi}{2}\right)\right)$ or a maximally entangled state $(\alpha=0)$. Let $\xi=\frac{\omega t}{\hbar}$. Then the time dependent, optimally evolving state, the concomitant entanglement, and its time average are respectively given by,

$$
\begin{aligned}
|\Psi(\xi, \alpha)\rangle & =\cos \xi|00\rangle+\sin \xi \frac{\cos \alpha}{\sqrt{2}}[|01\rangle+|10\rangle] \\
& +\sin \xi \sin \alpha|11\rangle, \\
\mathcal{E}(\xi, \alpha) & =\left(\cos (\alpha)^{2} \sin (\xi)^{2}-\sin (\alpha) \sin (2 \xi)\right)^{2} \\
\langle\mathcal{E}(\alpha)\rangle & =\frac{3}{8} \cos (\alpha)^{4}-\frac{2}{\pi} \cos (\alpha)^{2} \sin (\alpha) \\
& +\frac{1}{2} \sin (\alpha)^{2} .
\end{aligned}
$$

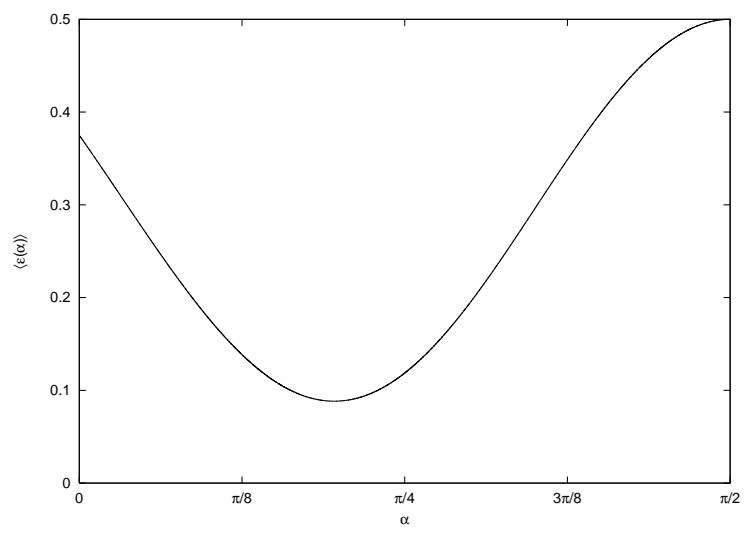

FIG. 1: Plot of $\langle\mathcal{E}(\alpha)\rangle$ as a function of $\alpha, \alpha \in\left[0, \frac{\pi}{2}\right]$.

Figure 1 is a plot of the time averaged entanglement $\langle\mathcal{E}(\alpha)\rangle$ as a function of the parameter $\alpha$. For $|00\rangle \rightarrow|11\rangle$ $\left(\alpha=\frac{\pi}{2}\right)$ we have the optimal time evolution of two qubits from an initial symmetric separable state into a final symmetric separable state orthogonal to the initial one. In this case we have $\langle\mathcal{E}\rangle=\frac{1}{2}$, which is the maximum time-average entanglement in this family of evolutions. On the other hand $|00\rangle \rightarrow \frac{1}{\sqrt{2}}[|01\rangle+|10\rangle]$ $(\alpha=0)$ constitutes an instance of the optimal evolution of a separable state into a symmetric maximally entangled state. In this case we have that $\langle\mathcal{E}\rangle=\frac{3}{8}$. Of particular interest is the minimum of the time-average entanglement within the family, which happens when $|00\rangle \rightarrow \frac{1}{\sqrt{3}}[|01\rangle+|10\rangle+|11\rangle]\left(\alpha=\arcsin \left(\frac{1}{\sqrt{3}}\right)\right)$ and gives $\langle\mathcal{E}\rangle=0.088298$.

(ii). This constitutes a particular instance of the optimal transformation of a symmetric maximally entangled state to another symmetric maximally entangled state. The time dependent entanglement and its average are, respectively,

$$
\mathcal{E}(t)=\cos ^{2}\left(\frac{\omega t}{\hbar}\right), \quad\langle\mathcal{E}\rangle=\frac{1}{2} .
$$

In this particular case the same amount of time-averaged entanglement is needed to transform a maximally entangled state into another maximally entangled state as is needed to transform a separable state into another separable state.

(iii) In this case the optimally evolving state is maximally entangled at all times, consequently giving $\mathcal{E}=1$.

\section{TYPICAL ENTANGLEMENT PROPERTIES OF TIME-OPTIMAL EVOLUTIONS OF TWO-QUBIT SYSTEMS}

Even when considering only two qubits and restricting $\left|\psi_{I}\right\rangle$ and $\left|\psi_{F}\right\rangle$ to be symmetric and orthogonal, the expression for $\langle\mathcal{E}\rangle$ becomes quite involved, thus making an analytic treatment of certain properties of the optimal evolution unpractical.

In order to explore the typical entanglement features of optimal evolutions in two-qubits systems we are going to generate random pairs of symmetric (orthogonal) initial and final states,

$$
\begin{aligned}
& \left|\psi_{I}\right\rangle=c_{1}|00\rangle+c_{2} \frac{1}{\sqrt{2}}(|01\rangle+|10\rangle)+c_{3}|11\rangle, \\
& \left|\psi_{F}\right\rangle=d_{1}|00\rangle+d_{2} \frac{1}{\sqrt{2}}(|01\rangle+|10\rangle)+d_{3}|11\rangle,
\end{aligned}
$$

with $\sum_{i}\left|c_{i}^{2}\right|=\sum_{i}\left|d_{i}^{2}\right|=1$ and $\sum_{i} c_{i} d_{i}^{*}=0$. We treat the case of symmetrical states separately because, as we shall presently see, in this case the necessity of entanglement to implement time-optimal dynamics is somewhat stronger than in the general case. However, an analysis similar to the one performed here for symmetrical states can be implemented for other particular families of states. We are going to consider the statistical distribution, and the mean and minimum values of the time averaged entanglement $\langle\mathcal{E}\rangle$ associated with the time-optimal evolutions connecting the pairs of states $\left|\psi_{I}\right\rangle$ and $\left|\psi_{F}\right\rangle$. Interpreting the triplets $\left(c_{1}, c_{2}, c_{3}\right)$ and $\left(d_{1}, d_{2}, d_{3}\right)$ as vectors in a three dimensional Hilbert space, we are going to generate the random states (12) by applying random $3 \times 3$ 
unitary matrices $U$ to the vectors $(1,0,0)$ and $(0,1,0)$. The random matrices $U$ are generated uniformly according to the Haar measure (see [13] and references therein). The corresponding probability distribution for the time averaged entanglement $\langle\mathcal{E}\rangle$ is depicted in Figure 2 (solid line). This distribution, describing the (fractional) volume of state-space associated with different values of $\langle\mathcal{E}\rangle$, has a lower cut-off at $\langle\mathcal{E}\rangle_{\min }=0.03415330$, meaning that, in order to implement a time-optimal evolution between symmetric two-qubit states a finite, minimum amount of entanglement is needed. Such an evolution cannot be implemented without entanglement. On the other hand, there are time-optimal evolutions between symmetric states exhibiting a maximum time-averaged entanglement $\langle\mathcal{E}\rangle_{\max }=1.0$. The mean value (over all possible optimal evolutions connecting symmetric states of two qubits is $\langle\mathcal{E}\rangle_{\text {mean }}=0.5$.

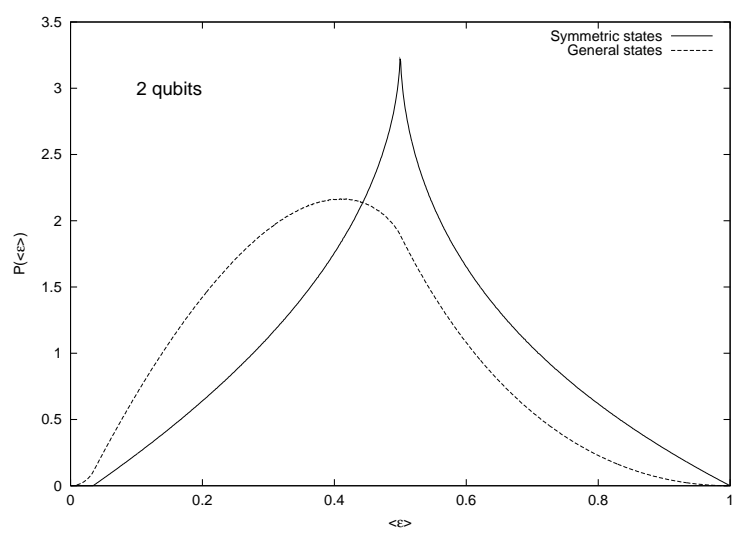

FIG. 2: Plot of the probability density function of the time averaged entanglement $\langle\mathcal{E}\rangle$ associated with symmetric and general two-qubit states.

We have also performed a similar calculation for time-optimal evolutions connecting two general (that is, not necessarily symmetrical) normalized and orthogonal states of two qubits, $\left|\psi_{I}\right\rangle=a_{1}|00\rangle+a_{2}|01\rangle+a_{3}|10\rangle+$ $a_{4}|11\rangle$ and $\left|\psi_{F}\right\rangle=b_{1}|00\rangle+b_{2}|01\rangle+b_{3}|10\rangle+b_{4}|11\rangle$. The vectors $\left(a_{1}, a_{2}, a_{3}, a_{4}\right)$ and $\left(b_{1}, b_{2}, b_{3}, b_{4}\right)$ were obtained by acting with random unitary matrices $U$ (generated uniformily according to the Haar measure) upon the vectors $(1,0,0,0)$ and $(0,1,0,0)$, respectively. The corresponding probability distribution for the $\langle\mathcal{E}\rangle$-values is shown in Figure 2 (dashed line). In this case there are optimal evolutions with arbitrarily small values of the time averaged entanglement. However, the probability density $P(\langle\mathcal{E}\rangle)$ approaches zero as $\langle\mathcal{E}\rangle \rightarrow 0$. Indeed, as we have already shown, the only optimal evolutions with $\langle\mathcal{E}\rangle=0$ are those of the form (8) where one of the subsystems does not evolve. It can be clearly appreciated in Figure 2 that, as a general trend, more entanglement is involved in optimal evolutions between symmetric states than the one involved in general optimal evolutions (see also Table I).

\section{SYSTEMS OF HIGHER DIMENSIONALITY}

We have also considered the connection between entanglement and brachistochrone quantum evolution for twoqutrit and three-qubit systems (as entanglement measure for three-qubit states we used the average bi-partite entanglement associated with the three bi-partitions of the system into a qubit and a two-qubit subsystem). The corresponding probability densities for the time averaged entanglement associated with brachistochrone evolutions connecting random orthogonal states are depicted in Figure 3. It transpires from this Figure that, both for twoqutrit and three-qubit systems, a considerable amount of entanglement is involved in typical brachistochrone evolutions.
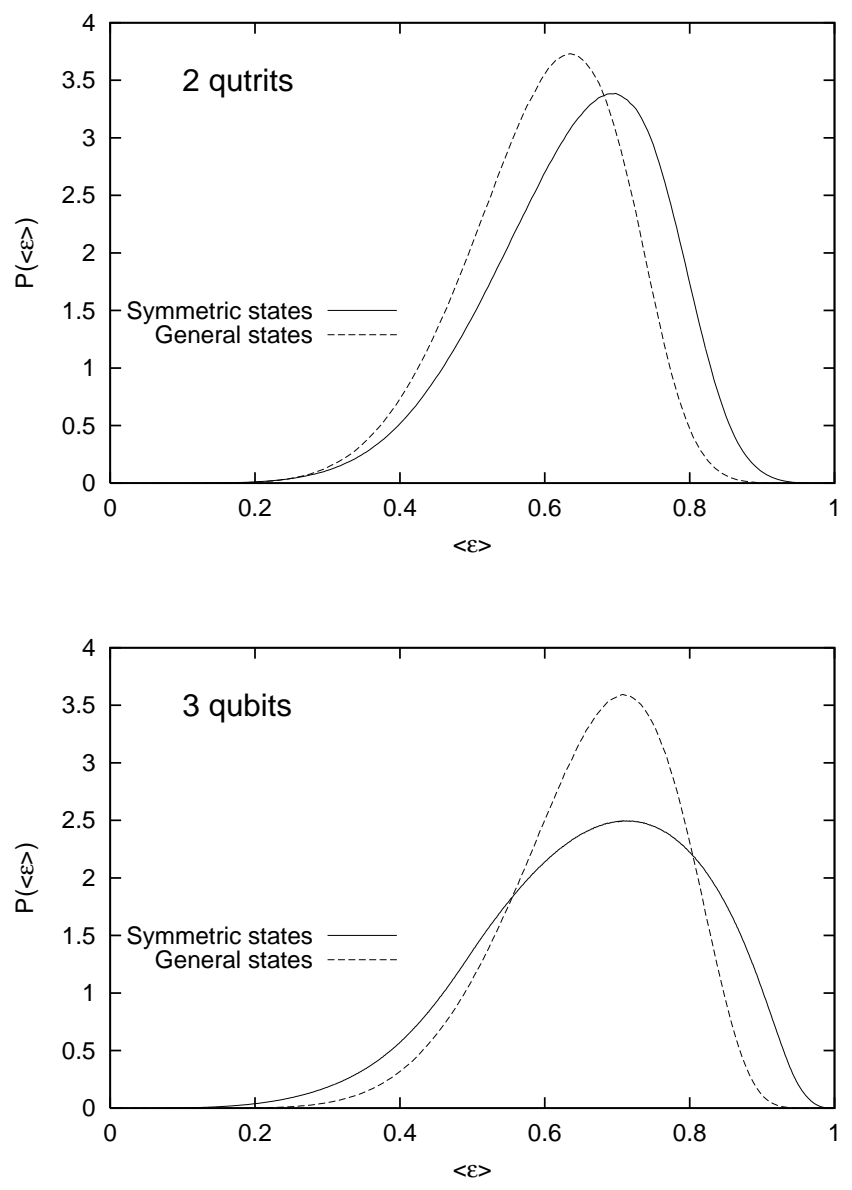

FIG. 3: Plot of the probability density function of the time averaged entanglement associated with time optimal quantum evolutions between symmetric and general orthogonal states of two-qutrit and three-qubit states.

The minimum, maximum, mean and likeliest values of the time averaged entanglement associated with brachistochrone evolutions between orthogonal symmetric $(\mathrm{S})$ and general $(\mathrm{G})$ states (for two-qubit, three-qubit and 
two-qutrit systems) are given in Table 1 .

\begin{tabular}{|c|c|c|c|c|c|c|}
\hline & \multicolumn{2}{|c|}{2 qubits } & \multicolumn{2}{c|}{3 qubits } & \multicolumn{2}{c|}{2 qutrits } \\
\hline & $\mathrm{S}$ & $\mathrm{G}$ & $\mathrm{S}$ & $\mathrm{G}$ & $\mathrm{S}$ & $\mathrm{G}$ \\
\hline$E_{\text {min }}$ & 0.0342 & 0.0000 & 0.0304 & 0.0000 & 0.0256 & 0.0000 \\
\hline$E_{\text {max }}$ & 1.0000 & 1.0000 & 1.0000 & 1.0000 & 0.9914 & 0.9915 \\
\hline$E_{\text {mean }}$ & 0.5000 & 0.4000 & 0.6667 & 0.6667 & 0.6429 & 0.5999 \\
\hline$E_{\text {likeliest }}$ & 0.50 & 0.41 & 0.71 & 0.71 & 0.69 & 0.64 \\
\hline
\end{tabular}

TABLE I: Minimum, maximum, mean and likeliest timeaveraged entanglement associated with brachistochrone evolutions of systems of 2 qubits, 3 qubits, and 2 qutrits.

\section{CONCLUSIONS}

We have investigated the role of entanglement in timeoptimal (brachistochrone) evolution of composite quantum systems. We proved that, except for trivial cases in which only one of the subsystems is actually evolving, brachistochrone quantum evolution between orthogonal states cannot be implemented without entanglement. We studied in detail, for two-qubits, three-qubits and two-qutrits systems, the amount of entanglement (as measured by the time averaged entanglement) involved in typical brachistochrone evolutions connecting orthogonal initial and final states. In all cases we found that a considerable amount of entanglement is needed in order to implement brachistochrone evolutions. The present approach to the study of the connection between entanglement and quantum time-optimal evolution may be regarded as "global" in the sense that (for a given system) it is not based upon the separate analysis of the the dynamics generated by different possible Hamiltonians. If we consider, for instance, a two-qubits system, the global approach shows that, in general, entanglement is a necessary resource to implement optimal quantum evolutions. Previous approaches, on the contrary, would require a separate treatment of each different Hamiltonian of the system at hand (say, a two-qubit system).

The two alluded strategies, the "global" and the "Hamiltonian-specific" one, actually complement each other. The global approach, based upon the brachistochrone evolution, establishes in a general and unified way that there is a connection between entanglement and optimal quantum evolution between orthogonal pure states: considering at the same time (for a given system) all possible optimum quantum evolutions it is seen that most of them involve a considerable amount of entanglement. On the other hand, the Hamiltonian-specific treatment permits, for each specific quantum Hamiltonian, a more detailed analysis of the aforementioned connection 6. 7, 8, 9, 10].

Summing up, in the present work we have established a definite connection between entanglement and brachistochrone evolutions connecting pairs of pure orthogonal states. Arguably, time-optimal evolutions between pure and orthogonal states are (at least from the conceptual point of view) the most important ones, since the initial and the final state are fully distinguishable and the alluded evolution can be identified with one elementary information-processing step [11]. However, it is possible to incorporate within the present approach brachistochrone evolutions connecting non-orthogonal pure states, since these evolutions are perfectly well defined [3]. Moreover, it would also be possible to explore the case of optimal time-evolution between mixed states, using the techniques advanced in [5]. We plan to investigate these extensions of the ideas advanced here in a future contribution.

Acknowledgements. We would like to thank the anonimous referees for their useful suggestions that helped to clarify and improve the present contribution. The financial assistance of the National Research Foundation (NRF; South African Agency) towards this research is hereby acknowledged. Opinions expressed and conclusions arrived at, are those of the authors and are not necessarily to be attributed to the NRF. This work was partially supported by the MEC grant FIS200502796 (Spain) and FEDER (EU), by the Government of Balearic Islands, and by CONICET (Argentine Agency). A. Borrás acknowledges support from FPU grant AP2004-2962 (MEC-Spain).
[1] Mach E., The Science of Mechanics (Open Court, La Salle) 1989.

[2] Carlini A., Hosoya A., Koike T., and Okudaira Y., Phys. Rev. Lett., 96 (2006) 060503.

[3] Brody D. C. and Hook D. W., J. Phys. A: Math. Gen., 39 (2006) L167; J. Phys. A: Math. Theor., 40 (2007) 10949 (Corrigendum).

[4] Bender C.M., Brody D.C., Jones H.F., and Meister B.K., Phys. Rev. Lett., 98 (2007) 040403.

[5] Carlini A., Hosoya A., Koike T., and Okudaira Y., quant-ph/0703047
[6] Giovannetti V., Lloyd S. and Maccone L., Europhys. Lett., 62 (2003) 615.

[7] Giovannetti V., Lloyd S. and Maccone L. Phys. Rev. A, 67 (2003) 052109.

[8] Batle J., Casas M., Plastino A. and Plastino A. R., Phys. Rev. A, 72 (2005) 032337.

[9] Borras A., Casas M., Plastino A. R. and Plastino A. Phys. Rev. A, 74 (2006) 022326.

[10] Zander C., Plastino A. R., Plastino A. and Casas M., J. Phys. A: Math. Gen., 40 (2007) 2861.

[11] Margolus N. and Levitin L. B., Physica D, 120 (1998) 
188.

[12] Verhulst T. and Naudts J., J. Phys. A: Math. Gen., 40 (2007) 2475.
[13] Batle J., Casas M., Plastino A. R. and Plastino A., Phys. Lett. A, 296 (2002) 251. 